\title{
Application of ray-tracing through the high resolution numerical weather model HIRLAM for the analysis of European VLBI
}

\author{
Susana Garcia-Espada ${ }^{a}$, Rüdiger Haas ${ }^{b}$ and Francisco Colomer ${ }^{c}$ \\ ${ }^{a}$ National Geographic Institute (IGN) \\ Apartado 148 E-19080 Yebes (Guadalajara), Spain \\ ${ }^{b}$ Chalmers University of Technology, \\ Department of Earth and Space Science, \\ Onsala Space Observatory, \\ SE-439 92 Onsala, Sweden \\ ${ }^{c}$ National Geographic Institute (IGN) \\ Calle General Ibañez de Ibero 3, E-28003 Madrid, Spain \\ E-mail: s.gespada@oan.es, rudiger.haas@chalmers.se, f.colomer@oan.es
}

\begin{abstract}
The tropospheric effects caused by the neutral atmosphere are an important limitation on the precision of results obtained using space geodetic techniques like VLBI and GPS. In recent years numerical weather models (NWM) have been applied to improve mapping functions which are used for tropospheric delay modeling in VLBI and GPS data analyses. A method for a direct calculation of the slant delay via raytracing through the 3D-VAR HIgh Resolution Limited Area Model (HIRLAM) for numerical weather prediction (NWP) is developed and applied to the analysis of geodetic European VLBI data. The advantages of the HIRLAM model are the high spatial resolution $\left(0.2^{\circ} \times 0.2^{\circ}\right)$ and the high temporal resolution in prediction mode ( 3 hours). As a result of using raytrace delays for the VLBI data analysis, estimated zenith wet delays (ZWD) are smaller than those using the standard Niell Mapping Function (NMF). Baseline repeatability improves with the HIRLAM approach for cut-off angles higher than $19^{\circ}$.
\end{abstract}

10th European VLBI Network Symposium and EVN Users Meeting: VLBI and the new generation of radio arrays

September 20-24, 2010

Manchester Uk 


\section{Introduction to the HIRLAM 3D-Var Numerical Weather Prediction Model}

In recent years, regional-scale NWMs have improved in terms of accuracy and precision. Thus it appears to be reasonable to calculate slant delays by ray-tracing through these NWMs and to apply these slant delays as external information for the analysis of space geodetic data. The HIRLAM project ([1]) has been established in order to provide the best available operational short-range forecasting system for the National Meterological Services in Denmark, Finland, Iceland, Ireland, Netherlands, Norway, Spain and Sweden. Meteo-France has a research cooperation agreement with HIRLAM. The HIRLAM system is a complete NWP system including data assimilation with analysis of conventional or non-conventional observations and a limited area forecasting model with a comprehensive set of physical parametrization. The forecast model is a limited area model with a boundary relaxation scheme. The model exists both in a grid-point version and in a spectral version. Initial and boundary conditions are taken from European Centre for Medium Range Weather Forecast (ECMWF). The HIRLAM model is a synoptic scale model which means it is displaying conditions simultaneously over a broad area. It is a numerical short-range $(<48 \mathrm{~h})$ weather forecasting system. The most used version is the hydrostatic grid point model. The advantage of the HIRLAM model are its high spatial resolution $(22 \mathrm{~km}$ to $5 \mathrm{~km}$ horizontally, 16 to 60 levels vertically) and high temporal resolution (6 hours assimilation data and analysis and prediction at 00h, 06h, 12h, 18h; 3 hours cycle also available).

\section{Application for the analysis of European VLBI}

We applied ray-tracing through the high resolution numerical weather model HIRLAM for the analysis of 15 geodetic European VLBI experiments from EURO75 (22 ${ }^{\text {nd }}$ March 2005) to EURO89 ( $3^{\text {rd }}$ September 2007) ([2]). We used HIRLAM files with $22 \mathrm{~km}$ horizontal resolution, 40 vertical levels and 6 hours time resolution $(00 \mathrm{~h}, 06 \mathrm{~h}, 12 \mathrm{~h}, 18 \mathrm{~h})$. Twelve different stations were involved: Crimea in Ukraine, DSS65a in Spain; Matera, Noto, and Medicina in Italy; Metsähovi in Finland; Ny-Ålesund in Norway; Onsala in Sweden; Svetloe and Zelenchukskaya in Russia; and Wettzell and Efflsberg in Germany. Badary station in Russia was also involved in EURO87 but it is not included in HIRLAM grid so we did not use its data. For each site and time epoch we generated profiles from the grid model with pressure, temperature and relative humidity for 40 vertical levels. We interpolated between the 4 nearest points around the site.

For a first approach we used 'The Davis/Herring/Niell Raytrace program' ([3]) to calculate the geometric, wet and dry delays. This program uses pressure, temperature and relative humidity profiles at a specified starting height above sea level to calculate path delay through the atmosphere. It is based on ray-tracing in a one-dimensional plane depending on the angle of elevation of the source observed so different azimuth values do not produce different output values for the same elevation. We used the SOLVE ([4]) software package to analyze the geodetic European VLBI data and to make a comparison between using the Niell Mapping Function (NMF) as a standard atmosphere, and ray-tracing through HIRLAM approach as a priori values for the atmosphere parameters.

Figure 1 shows examples of time series of estimated zenith wet delays (ZWD) for Onsala and Wettzell using NMF and using delays calculated with ray-tracing through HIRLAM as apriori values. In all cases the estimated ZWD are smaller and closer to zero using the HIRLAM approach. 
It means that the a priori knowledge about the atmosphere parameters is better in the HIRLAM approach. The variability of the estimated ZWD is also reduced using ray-tracing through HIRLAM compared to NMF.
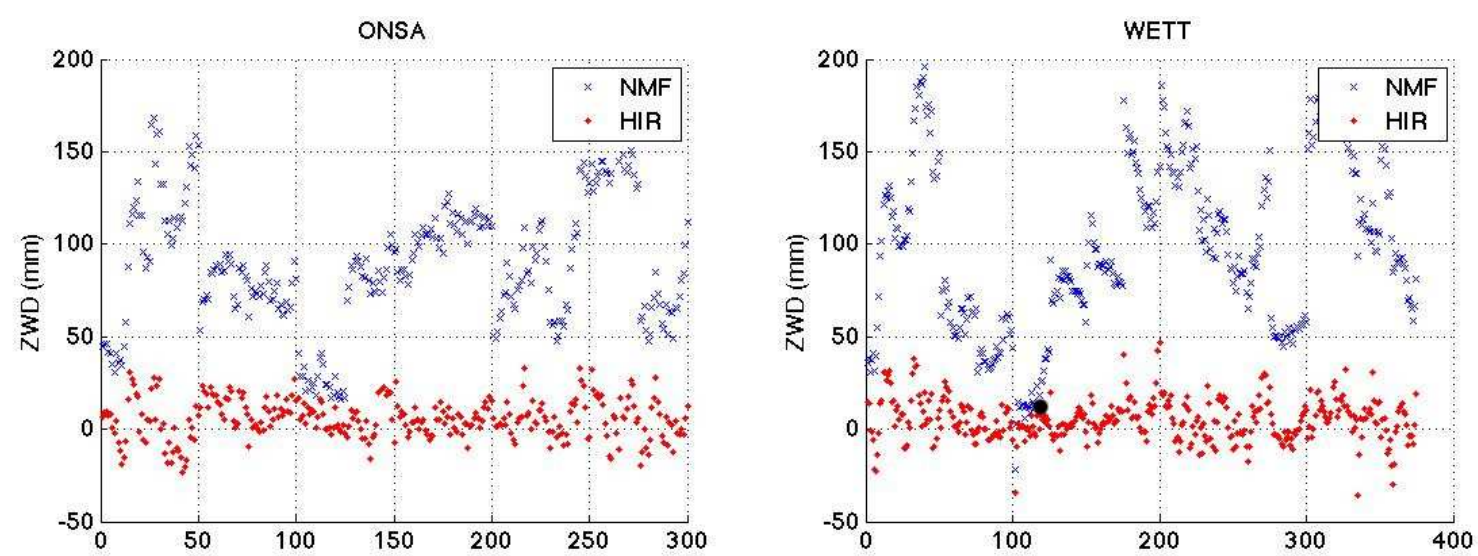

Figure 1: Estimated zenith wet delays time series for Onsala and Wettzell. Crosses show estimated ZWD using NMF, we see higher values and higher variability. Diamonds show estimated ZWD using ray-tracing through HIRLAM as apriori values, we see values closer to zero and lower variability.

Figure 2 shows baseline length repeability in terms of weighted root mean-square (wrms) as a function of baseline length, for cut-off angles of $10^{\circ}$ (left) and $19^{\circ}$ (right). The repeatability improves using ray-tracing through HIRLAM, compared with NMF, for a cut-off angle higher than $19^{\circ}$. Table 1 shows the rates and offsets of the linear regression fits to the wrms values as a function of baseline length for cut-off angles of $5^{\circ}, 10^{\circ}$ and $19^{\circ}$.

Currently we are working on a 4-D ray-tracing program, fully elevation and azimuth dependent for each observation, using a combination of HIRLAM analysis and forecast profiles to improve the temporal resolution (3h).
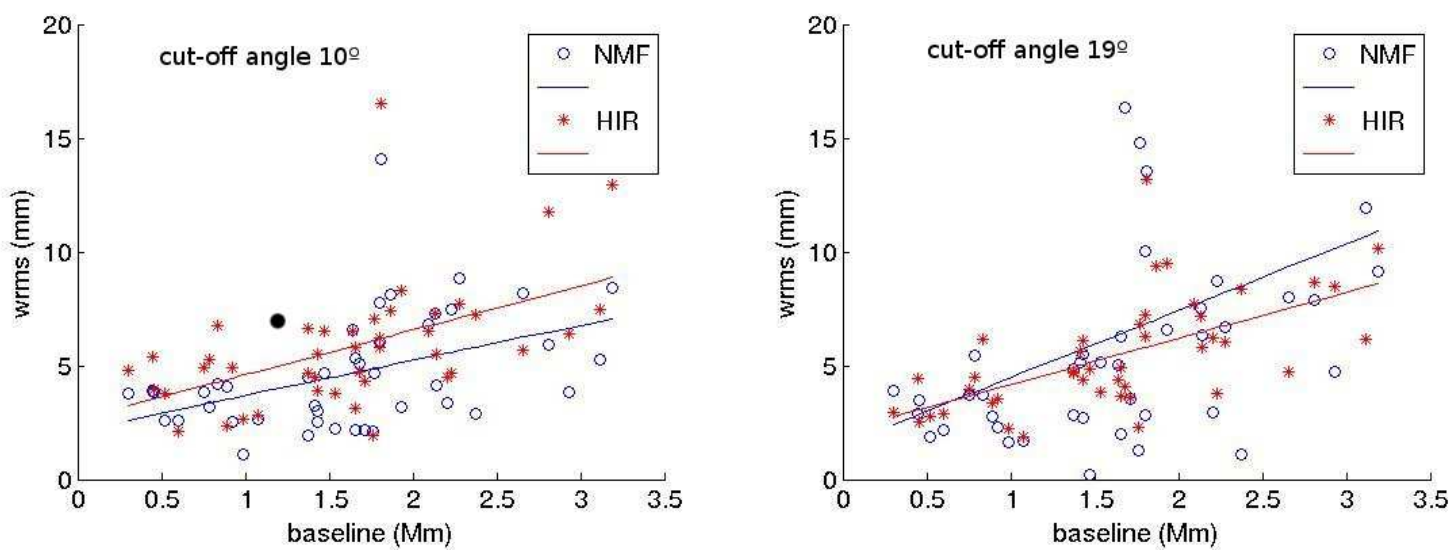

Figure 2: Baseline repeability for all baselines and different elevation cut-off angles. Weighted root meansquare (wrms) values are plotted against baseline length. 
Table 1: Baseline repeability rates and offsets

\begin{tabular}{|c|c|c|c|c|c|c|}
\hline \multirow{2}{*}{} & \multicolumn{2}{|c|}{ Cut-off angle $5^{\circ}$} & \multicolumn{2}{c|}{ Cut-off angle $10^{\circ}$} & \multicolumn{2}{c|}{ Cut-off angle $19^{\circ}$} \\
\cline { 2 - 7 } & rate & offset & rate & offset & rate & offset \\
\hline NMF & $1.14 \mathrm{ppb}$ & $2.6 \mathrm{~mm}$ & $1.54 \mathrm{ppb}$ & $2.1 \mathrm{~mm}$ & $2.93 \mathrm{ppb}$ & $1.5 \mathrm{~mm}$ \\
\hline HIRLAM & $2.04 \mathrm{ppb}$ & $2.9 \mathrm{~mm}$ & $1.95 \mathrm{ppb}$ & $2.6 \mathrm{~mm}$ & $2.03 \mathrm{ppb}$ & $2.1 \mathrm{~mm}$ \\
\hline
\end{tabular}

\section{References}

[1] Synoptic scale model HIRLAM, http://hirlam.org/

[2] Garcia-Espada, S., Haas, R., and Colomer, F., Application of ray-tracing through the high resolution numerical weather model HIRLAM for the analysis of European VLBI, In: 6th IVS General Meeting Proceedings, Hobart, Australia, 2010. In press.

[3] Davis, J.L., Herring, T.A.H. and Niell, A.E., The Davis/Herring/Niell Raytrace program, 1987-1989

[4] Ma, C., Clark T. A., Ryan J. W., Herring T. A., Shapiro I. I., Corey B. E., Hinteregger H. F., Rogers A. E., Whitney A. R., Knight C. A., Lundqvist G. L., Shaffer D. B., Vandenberg N. R., Pigg J. C., Schupler B. R. and Ronnang B. O., Radio-source positions from VLBI, Astron. J., 92, 1020-1029, 1986. 